\title{
O jogo I-AI3: um protótipo em desenvolvimento
}

\author{
Susana Cristina dos Reis ${ }^{1}$, Frederico Menine Schaf ${ }^{1}$, Andréia Oliveira ${ }^{1}$, Adilson Fernandes Gomes ${ }^{2}$, \\ Rosângela Segala de Souza $^{3}$ \\ ${ }^{1}$ Professores da Universidade Federal de Santa Maria - Núcleo de Tecnologias Educacionais \\ (NTE/UFSM) - CEP: 97105-900 - Santa Maria - RS - Brasil \\ ${ }^{2}$ Aluno do Curso de Letras/Inglês - Iniciação Científica - Universidade Federal de Santa Maria (UFSM) \\ ${ }^{3}$ Professora no Instituto Federal Farroupilha (IFF), Campus São Vicente do Sul - RS - Brasil

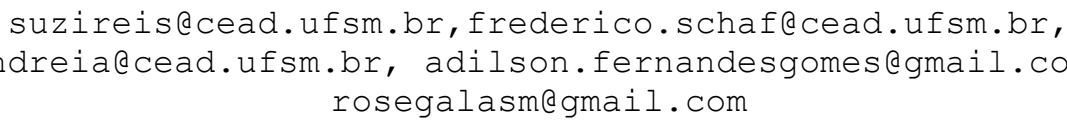

Resumo. Este artigo reporta a experiência de construção de um projeto de jogo em ambiente imersivo, envolvendo as áreas de Artes Visuais, Engenharia, Direito Ambiental e Língua Inglesa (LI). Esse protótipo usa ambientes virtuais de aprendizagem e a tecnologia OpenSim para criação do design do jogo e das atividades em LI. Os resultados sugerem que a construção das fases do jogo, bem como os desafios que envolvem as atividades em LI e os conhecimentos interdisciplinares exigem a formação de uma equipe que seja capaz de articular pressupostos teórico-metodológicos sobre jogos e conhecimentos tecnológicos, para garantir o desenvolvimento dos princípios de um bom jogo. Resultados dessa experiência serão apresentados no evento.

\begin{abstract}
This article reports an experience of constructing an immersing learning game, involving different areas such as Arts, Engineering, Environmental Law and English as a Foreign Language. This project is using virtual worlds and a learning management systems, OpenSim to propose a challenging prototype of an English Game. The results suggest that the development of phases in a game, as well as the challenges which involve activities in a Foreign Language and interdisciplinary knowledge requires the formation of a work-team that is able to articulate theoretical and methodological awareness about serious games, as well as technological skills to ensure good game development principles. Results about this experience will be shown at the event.
\end{abstract}

\section{Introdução}

$\mathrm{O}$ crescimento da Internet tem ocasionado o surgimento de novos paradigmas e possibilidades de ampliação dos processos de ensino-aprendizagem por meio de tecnologias digitais, especialmente para a prática de e-learning com o uso de realidades virtuais. Em particular, por meio da Internet, pessoas geograficamente dispersas podem acessar remotamente materiais didáticos elaborados em diferentes plataformas, para realizar atividades colaborativas e estabelecer interações, que venham reduzir alguns dos efeitos negativos geralmente gerados em cursos a distância.

Tendo em vista desenvolver projetos que incluam tecnologias interativas e princípios de jogos sérios (ou serious games), um dos projetos em desenvolvimento no LabInter - Laboratório Interdisciplinar Interativo - [Oliveira, Schaf e Reis 2012], "Jogos em AI3" - Ambientes Imersivos Interativos Interdisciplinares em 3D - será discutido. Assim, neste relato descrevemos a experiência de construção interdisciplinar do ambiente do jogo de Inglês em AI3, denominado I-AI3. Esse projeto faz uso de 
ambientes virtuais de ensino-aprendizagem e ambientes imersivos em 3D para ensinar conteúdos de língua inglesa e de educação ambiental.

Este artigo está dividido em três seções, sendo a primeira a revisão da literatura sobre interação e colaboração nos processos de ensino-aprendizagem em ambientes virtuais imersivos; a segunda discute sobre as tecnologias utilizadas no desenvolvimento do protótipo do jogo e, por fim, na terceira, propomos as considerações finais.

\section{Interação e Colaboração em Processos de Ensino-Aprendizagem com Tecnologias Digitais}

Evoluções recentes sobre o uso de redes sociais, ambientes virtuais, tecnologias Web e mundos virtuais tridimensionais na educação têm motivado a inserção de diferentes tecnologias nas práticas de ensino e de aprendizagem. Com isso, sucessivas possibilidades inovadoras, implementações e construção de cenários híbridos têm emergido, simulando situações que podem gerar aprendizagem de diferentes conteúdos.

Nesse panorama, parece importante desenvolver projetos que investiguem como melhorar, significativamente, a aprendizagem de conteúdos por meio de experiências interativas e colaborativas, envolvendo tecnologias digitais. No entanto, esse desafio tem levado alguns pesquisadores a reconhecerem que este não é apenas um desafio tecnológico, mas também organizacional e social [Grudin 1994; Kaptelinin 1996].

Independentemente da ênfase teórica adotada sobre aprendizagem colaborativa (construtivismo social, por exemplo), pesquisas indicam que alunos aprendem de forma mais eficaz quando inseridos em contextos que geram interações colaborativas, a partir da troca de conhecimentos com outros pares [Vygotsky 1998; Kreijns, Kirschner, Jochems 2003; Reis 2004]. Essa interação por meio de atividades colaborativas pode desenvolver a auto-crítica (reflexão) e impulsionar a construção do conhecimento. Tendo em vista esses pressupostos, não é difícil prever Ambientes Computacionais com Suporte a Colaboração (ACSC) na comunidade científicas, que podem ser aplicados no campo de e-learning.

Nesse sentido, as Tecnologias de Informação e Comunicação (TIC) podem promover a colaboração e a interatividade se houver intencionalidade para este modo de interrelações sociais [Oliveira, Fonseca e Biazus 2007]. Se for esse o objetivo, a inserção desses recursos pode provocar situações instáveis, orientando a construção do conhecimento, em vez de acumulação de dados. No entanto, é preciso tomar cuidado com a falsa neutralidade sobre a interatividade, como que se tudo o que é interativo fosse bom para o processo de aprendizagem.

A interatividade, segundo Massumi (2008), não é neutra no que diz respeito ao poder. Na verdade, entre os mais odiosos regimes de poder são os que impõem o uso imperativo para participar, especialmente, se o imperativo é "realmente" ou "autenticamente" expressar-se. Nesse caso, você (o sujeito) está constantemente "interpelado" [Massumi 2008]. Assim, buscamos pensar em projetos em que a interação acontece além das ações e das reações simples, em que o interator (ator da interação) possa refletir sobre abordagens sociais e computacionais.

De acordo com Piaget (1962) e Vygotsky (1998), o conhecimento é produzido em processos interativos. Para Piaget, acontece em um processo de assimilação e de 
acomodação por meio de saltos e situações de desequilíbrios; para Vygotsky ocorre em um processo de aprendizagem por meio da interação especializada. Para saber algo, devemos interagir com objetos e sujeitos. Portanto, o processo de aprendizagem acontece em uma mudança estrutural, produzida em um jogo de assimilação e de acomodação por meio da interação coletiva baseada na cooperação.

Esses pressupostos levam a crer que os projetos desenvolvidos no LabInter, como o exemplo do projeto I-AI3 conectam algumas das discussões propostas neste artigo.

\subsection{Ferramentas para o desenvolvimento de ambientes imersivos}

Ambientes Virtuais de Ensino-Aprendizagem (AVEA) ou Sistemas de Gerenciamento de Aprendizagem/Curso (do inglês LMS/CMS) são as ferramentas mais comuns utilizadas para organizar a mídia digital com objetos de ensino e aprendizagem na $W e b$. A implementação mais comum e livre dos AVEA, atualmente, parece ser o Moodle [Dougiamas e Taylor 2003] em função da grande quantidade de hospedagens de cursos e-learning.

O Moodle é um sistema de gerenciamento de código aberto que é baseado na pedagogia construtivista social. A maioria das implementações de AVEA serve como ajuda para professores explorarem ferramentas gráficas para criar, hospedar e vincular seus materiais didáticos. Esse ambiente também fornece controle de usuário e de informações de $\log$ que ajudam a rastrear as interações dos usuários, tanto com o ambiente virtual quanto com outros usuários. Embora os softwares possam ser concebidos para gerar aproximações sociais ou com objetivos específicos, ainda tais recursos, muitas vezes, são insuficientes para apoiar a colaboração e para suportar a interação social sem algum tipo de partilha ou coautoria.

Atualmente a possibilidade de criar mundos virtuais por meio de plataformas como o Second Life ou o Active Worlds - AWEDU - gera ambientes multiusuários (MUVE - Multi-User Virtual Environments), permitindo desenvolver conteúdos mais interativos, que apontam para uma solução mais lúdica e propícia para a interface de jogos. Essa associação, de AVEA e mundos virtuais, geralmente, cativa a atenção dos alunos para a produção do conhecimento [Gee 2004; Johnson 2005].

$\mathrm{Na}$ literatura recente, os ambientes virtuais que utilizam o jogo como interface em seu projeto, com outros fins, além do entretenimento, são denominados jogos sérios ou educativos (serious games). A maioria dos Jogos Multiusuários de RPG (MMORPG) são desenvolvidos para alcançar esse mundo virtual (principalmente, mundos de fantasia) por meio de interfaces imersivas.

Existem várias técnicas e equipamentos para alcançar diferentes níveis de imersão, tais como: monitores normais, monitores 3D, HMD, "cavernas" com múltiplas projeções, dispositivos de force feedback de interação, entre outros. A sensação de imersão mais comum é alcançada pelo uso de avatares em ambientes tridimensionais virtuais, mundos virtuais ou metaversos, dando ao usuário "a sensação de estar lá", mesmo que apenas virtualmente [Enlund 2001].

Usuários virtuais imersos, avatares, navegam por meio do(s) metaverso(s) para interagir com objetos virtuais ou outros avatares. Tal associação de ferramentas também 
já foi utilizada para o treinamento de engenheiros, como o proposto por Schaf, Paladini e Pereira (2012).

Focando nesses conceitos e tecnologias é que propomos o protótipo do jogo I$\mathrm{AI} 3$, conforme descreveremos na próxima seção.

\section{English Online 3D e o Jogo I-AI3: conectando pressupostos teórico- metodológicos}

Originalmente o English Online $3 D$ é um projeto de e-learning que tem por objetivo conectar alunos na Internet, de forma que estes se envolvam em atividades de linguagem por meio de diferentes gêneros digitais [Reis, 2004; 2005; 2006; 2010] e tecnologias imersivas com a finalidade de aprender a linguagem por meio da interação virtual.

$\mathrm{Na}$ literatura de ensino de línguas com base em gêneros [Bazerman 2005; Hyland 2007; Motta-Roth, Reis e Marschall 2007], pesquisadores sugerem que o ensino deve ser orientado por atividades que incluam gêneros discursivos/textuais que possibilitam a interação dos participantes por meio de diferentes práticas sociais, com vistas a desenvolver a aprendizagem por meio da prática e do uso da linguagem [Paiva 1999; 2010]. Assim, no curso English Online 3D, buscamos explorar gêneros e contextos que levem os alunos à resolução de problemas propostos por meio de atividades de língua inglesa.

Com o propósito de elaborar um jogo adequado às necessidades do curso English Online 3D, decidimos prever nas atividades do Projeto English Online 3D a prática da língua inglesa por meio de um jogo imersivo, que permitisse ao aluno discutir questões relacionadas à educação ambiental e aprimorar seu conhecimento de língua, dentro dessa temática. Desta forma, com o apoio da equipe do LabInter, iniciamos a construção do protótipo do jogo I-AI3 para ser usado pelos participantes do curso.

Tendo em vista desenvolver a aprendizagem crítica no ensino interdisciplinar, buscamos um suporte teórico que atendesse nossas expectativas, pois segundo Gee (2004) todo bom jogo tem por trás uma boa teoria de aprendizagem. Para isso, adotamos alguns princípios que orientam tanto a construção do protótipo quanto a aprendizagem de línguas, que são: a linguagem é uma prática social [Halliday 1989; Motta-Roth 2008], portanto, precisamos possibilitar que os participantes interajam por meio da linguagem para desenvolver diferentes habilidades linguísticas através do jogo; os participantes devem buscar desenvolver multiletramentos [Motta-Roth e Hendges 2010] e, os conteúdos (linguísticos ou de diferentes áreas) devem ser desafiantes [Gee 2004; Johnson 2005] e hipertextuais para gerar aprendizagem.

O protótipo do jogo I-AI3 está conectado a um mundo virtual em 3D (metaverso) e, para isso utilizamos o Open Simulator [OpenSim 2012] como hospedeiro, ou seja, seguindo a arquitetura EduWorlds [EduWorlds, 2012].

Começamos a desenvolver o I-AI3 como um espaço virtual para colaboração e construção interdisciplinar, conectando a aprendizagem de língua inglesa com a discussão de questões ecológicas em um único protótipo. No OpenSim, planejamos, até o presente momento, três fases em que os alunos podem se conectar para jogar e, ao mesmo tempo, aprender inglês. Os participantes são representados por avatares que 
usarão a língua inglesa para interagir uns com os outros, discutindo sobre questões ecológicas no âmbito urbano.

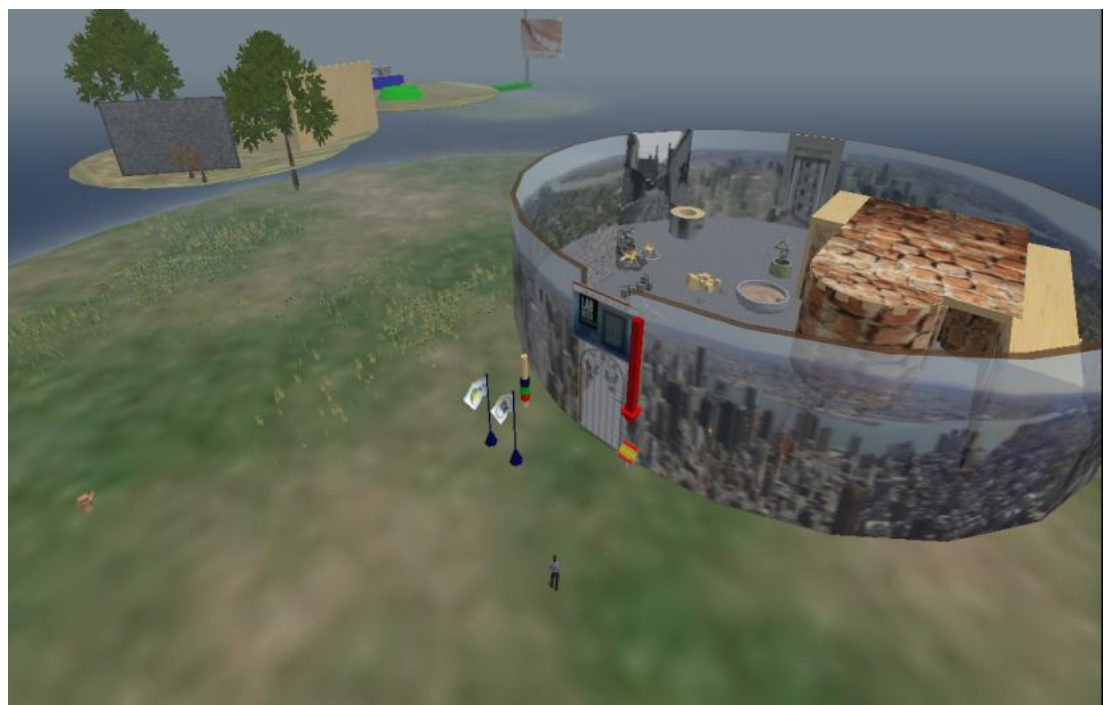

Figura 1. Etapa inicial do Jogo I-Al3

Para elaborar as atividades das três fases do protótipo de jogo imersivo I-AI3, desenvolvemos uma narrativa para o jogo que situa o participante em um determinado contexto e situação. Nesse ambiente, o participante assume uma identidade digital representada por um avatar, o qual é desafiado a tomar uma decisão para solucionar um problema proposto dentro do contexto social em que está inserido.

Na fase 1, o aluno inicia o jogo em uma praça da cidade, que está em situação degradante, com poluição visível em todo o lugar. Nessa fase, o participante deverá refletir sobre o cenário que está visualizando, bem como tomar uma atitude sobre o que pode fazer para melhorar aquele ambiente. Para isso, um tutor virtual encontra-se no local assumindo a identidade de uma ambientalista. Nessa situação, a ambientalista dará instruções para que o aluno encontre no cenário objetos, palavras ou sentenças em LI, que representam possíveis soluções para os problemas da praça.

De acordo com a solução desse problema, o participante terá uma recompensa que poderá ser um bônus ou um conhecimento linguístico, que lhe favorecerá na execução das atividades. Nessa perspectiva, atendemos aos fatores de satisfação que envolvem os participantes no ato de jogar [Johnson 2005], ou seja, o desafio, a tomada de decisão e a recompensa.

Buscamos desenvolver um ambiente virtual que possua características dialógicas, não lineares, e interativas para criar diferentes universos significativos. Para isso, é necessário produzir um jogo que faça uso de linguagens híbridas, conectando imagens, som e textos que tenham conexões significativas com o objetivo de aprendizagem. Assim, na fase 2 , os alunos são levados a vivenciar a prática de reciclagem, fazendo a coleta e a seleção de materiais que se encontram espalhados pela praça. Para cumprir o desafio proposto nessa fase, o aluno precisa tomar decisões e fazer associações entre conhecimentos linguísticos de LI e de educação ecológica. 


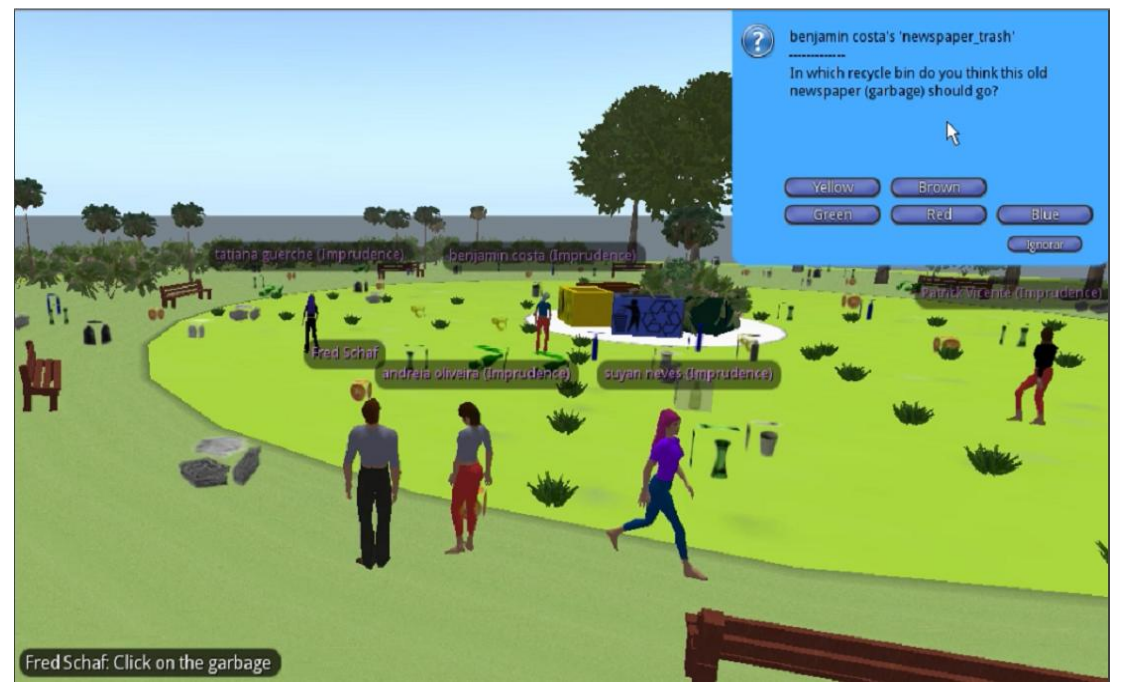

Figura 2. Fase do Jogo I-Al3

Para Bairon (2004), há atualmente na Internet muitas oportunidades para o desenvolvimento de metodologias hipermidiáticas, que permitem ir além da divisão de tarefas, que explorem apenas a leitura e a escrita, ou apenas a arte (imagem e som). Temos que considerar a multiplicidade de multimodalidades que se projetam em diferentes ambientes virtuais e que podem se tornar significativas para a aprendizagem de uma língua estrangeira. De acordo com Fonseca (2005, p. 127), "podemos supor que as potências de imagens digitalizadas instauram um novo regime semiótico em que o referente é anulado remetendo às imagens a si próprias".

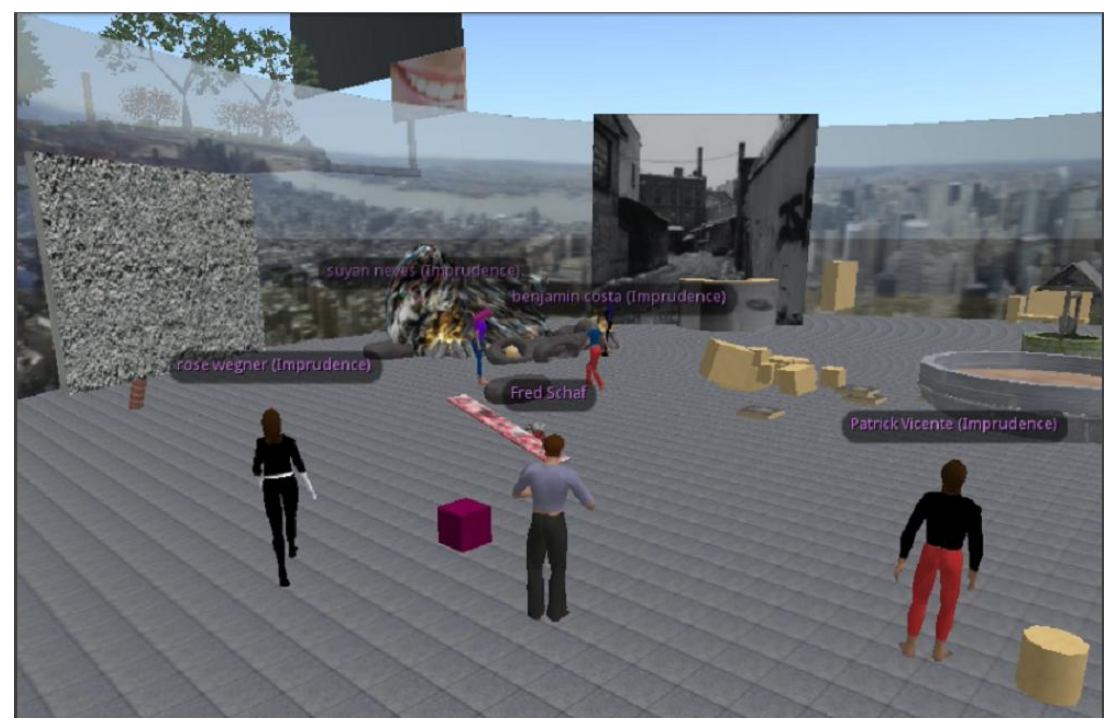

Figura 3. Fase do Jogo I-Al3

Além disso, almejamos desenvolver, por meio dos desafios propostos no jogo, novos letramentos, a partir da inserção dos participantes no uso de gêneros digitais, que possibilitem a aprendizagem dos alunos por meio de hipertextos e de situações hipermidiáticas, as quais simulem situações reais. Assim, na fase 3, projetamos inicialmente atividades que provocam os alunos a transformar a praça em um local ecologicamente correto, reconstruindo, por exemplo, objetos danificados e desenvolvendo atividades de jardinagem. 


\subsection{As dificuldades e conflitos encontrados na elaboração da proposta do protótipo do jogo imersivo I-AI3}

O processo interdisciplinar de elaboração do protótipo do jogo está envolvido por uma série de desafios que implicam em conquistas, dificuldades e conflitos, os quais podem ser de ordem teórico-metodológica, bem como do uso da tecnologia.

As dificuldades e os conflitos de ordem teórico-metodológica compreendem em fazer com que todos os membros da equipe fundamentem-se pelo mesmo suporte teórico e metodológico na elaboração do protótipo do jogo. Verificamos que as dificuldades geradas aconteciam por envolverem profissionais de diferentes áreas do conhecimento (Letras, Artes, Engenharia e Direito), tornando-se extremamente difícil estabelecer um princípio comum, que satisfizesse as exigências de todos. Como o projeto é um só, envolve muita negociação, pois cada profissional tem uma concepção de produção diferente, principalmente, quando estão envolvidas concepções teóricas, que dizem respeito às diferentes áreas disciplinares.

Para isso, optamos em fazer um levantamento teórico sobre o desenvolvimento de jogos imersivos, tanto em literatura produzida no Brasil, quanto no exterior, como uma alternativa para que todos os integrantes da equipe lessem sobre o mesmo assunto e pudessem discutir com mais propriedade sobre esse tema, visto que todos estão envolvidos na elaboração das atividades do projeto do jogo.

Ao elaborarmos atividades para e-learning devemos ter o cuidado de não usarmos a transposição de materiais didáticos utilizados em sala de aula convencional, mas sim criarmos um ambiente em que o aluno possa ter ao seu alcance diferentes formas de aprender. No jogo, em que ocorre aprendizagem e entretenimento, a dificuldade de estabelecer essa associação é um dos primeiros desafios enfrentados pela equipe, pois a aprendizagem deve ocorrer de forma sistematizada, mas sem a formalidade de um estudo tradicional, e também deve estimular o interesse do aprendiz de modo que ele siga até o final, superando os desafios do jogo, sem que esse se torne cansativo e enfadonho.

O conflito de ordem tecnológica ocorre tendo em vista que o protótipo I-AI3 está sendo desenvolvido na plataforma Open Simulator e nem todos os integrantes dominam e entendem o funcionamento dessa tecnologia. Com isso, ter a dimensão das possibilidades e das limitações do OpenSim é algo que envolve estudo e tempo, pois nem sempre o que é desejável ou planejado por um grupo de pesquisadores passa a ser executado, em função da falta de conhecimento da tecnologia. Por outro lado, esse processo de planejamento das atividades exige novas negociações e a reorganização das atividades planejadas.

De modo geral, as dificuldades e conflitos são benéficos para o projeto, pois, com isso, sentimos a necessidade de promover um ciclo de palestras semanais, no qual as decisões são negociadas, além de serem debatidos os textos selecionados para a leitura. É oportuno acrescentar, ainda, que esses textos envolvem todas as áreas do LabInter (Letras, Artes, Engenharia e Direito) em sistema rotativo de abordagens. As palestras são ministradas pelos integrantes dos grupos, visando um nivelamento do conhecimento de forma que todos os integrantes saibam o que cada grupo está lendo. Estabelecemos três conceitos-chave para guiar, de modo interdisciplinar, a equipe na 
elaboração do jogo: as concepções sobre o que são jogos educacionais, jogos sérios e gameart.

Os conflitos de ordem conceituais, tanto no que se refere ao processo ensinoaprendizagem quanto aos temas são elementos das discussões afloradas no planejamento, gerados pelos diferentes olhares de cada área possibilitando $o$ enriquecimento do projeto.

\section{Considerações Finais}

A tarefa de criar um ambiente interativo que envolva diferentes áreas do conhecimento ainda está em fase inicial, mas fornece subsídios para que os autores verifiquem que sem uma equipe multidisciplinar é impossível alcançar um projeto interdisciplinar como este que está sendo proposto. Os projetos do LabInter tentam compreender essa interação e agir como em um terreno fértil para novas ideias e projetos-piloto, que envolvam interatividade nas áreas de Artes e Tecnologia Aplicada à Educação.

A colaboração entre os pesquisadores do LabInter provou que diferentes áreas podem ser fundidas para atingir o máximo interesse e motivação dos alunos, por meio da elaboração de projetos como o do Jogo I-AI3. O desenvolvimento de técnicas de $e$ learning e novos ambientes tecnológicos são fundamentais. Para esses novos contextos, a palavra-chave é explorar diferentes formas de interação para extrair o melhor das tecnologias em todas as áreas de pesquisa. Como Guattari (1992, p. 16) coloca:

As evoluções tecnológicas, conjugadas a experimentações sociais desses novos domínios, são talvez capazes de nos fazer sair do período opressivo atual e de nos fazer entrar em uma era pós-mídia, caracterizada por uma reapropriação e uma re-singularização da utilização da mídia. (Acesso a bancos de dados, às videotecas, interatividade entre os protagonistas etc...).

O ambiente do Jogo I-AI3 proposto está apenas em fase de estudo. Contudo, verificamos que o protótipo apresenta algumas potencialidades, tais como: permitir a interação dos alunos em língua inglesa; possibilitar a construção do conhecimento sobre questões ecológicas, como por exemplo, os tipos de poluição, como reciclar produtos e os benefícios dessa reciclagem para o planeta Terra, além de estimular os alunos a refletirem sobre ações que poderão evitar a poluição.

Ao jogar, o aluno pode construir tanto um inventário linguístico, constituído por frases, sentenças, expressões e vocabulário relacionados ao tema meio ambiente, quanto fazer uso desse vocabulário ao interagir em LI com os outros participantes do jogo. Além disso, o I-AI3 possibilita, ainda, que os alunos joguem individualmente ou em equipe, com o objetivo de resolver as atividades propostas e desenvolver a consciência ecológica, a fim de que sejam capazes de agir não apenas no mundo virtual, mas também, no mundo real.

\section{Referências}

Bazerman, C. (2005) "Gêneros textuais, tipificação e interação". São Paulo: Cortez Editora, p. 9-68. 
Bairon, S. (2004) "Tendências da Linguagem Científica Contemporânea em expressividade digital: uma problematização". In Informática na Educação: Teoria \& Prática, Porto Alegre, v.7, n.2, p. 101-156, jul./dez. 2004.

Dougiamas, M.; Taylor, P. (2003) "MOODLE: using learning communities to create an open source course management system". In Proceedings of the World Conference on Educational Multimedia, Hypermedia and Telecommunications, Chesapeake, Australia, p.171-178.

Eduworlds. (2012) "Projeto Edutacional Worlds - buscando aperfeiçoar o ensino através do emprego de técnicas de experimentação remota e ambientes virtuais imersivos", http://www.ufsm.br/eduworlds/, Março.

Enlund, N. (2001) "Being Virtually There - Reality and Presence in Mediated Learning". In Proceedings of the 2001 International Conference on Telecommunications for Education and Training.

Fonseca, T. (2005) "Poéticas do virtual e os processos de subjetivação". In Informática na Educação: Teoria \& Prática - Revista do PGIE da UFRGS, v. 8, n. 1.

Gee, J. P. (2004) "What video games have to teach us about learning and literacy". New York: Palgrave Macmillan.

Guattari, F. (1992) "Restauração da Cidade Subjetiva", in Caosmose, Rio de Janeiro, ed. 34, p. 169-203.

Grudin, J. (1994) "Groupware and Social Dynamics: 8 Challenges for Developers". In Communications of ACM, v. 37, n. 1, p. 92-105, Califórnia: ACM Press.

Halliday, M. A. K. (1989) "Part A". In Halliday, M. A. K. \& Hasan, R. Language, context and text: Aspects of language in a social-semiotic perspective. Oxford: Oxford University, p. 3-49.

Hyland, K. (2007) “Genre pedagogy: language, literacy and L2 writing instruction". In Journal of Second Language Writing, v. 16, p.148-164.

Johnson, S. (2005) "Surpreendente! A televisão e o videogame nos tornam mais inteligentes". Rio de Janeiro: ed. Campus.

Kaptelinin, V. (1996) "Computer-mediated activity: functional organs in social and evelopmental contexts". In NARDI, B. (Org.), Context and consciousness: Activity Theory and human-computer interaction. Cambridge, Massachusetts: MIT Press, p. 45-68.

Kreijns, K.; Kirschner, P. A.; Jochems, W. (2003) "Identifying the pitfalls for social interaction in computer-supported collaborative learning environments: a review of the research". In Computers in Human Behavior, v. 19, p. 335-353.

Massumi, B. (2008) "The Thinking-Feeling of What Happens: A Semblance of a Conversation". In Inflexions Journal. http://www.senselab.ca/inflexions/pdf/Massumi.pdf, Maio.

Motta-Roth, D.; Reis e Marschall, D. (2007) "O Gênero página pessoal e o ensino de produção textual em Inglês”. In Júlio César de Araújo. (Org.) Internet e Ensino. 1 ed. Rio de Janeiro: Lucerna, p. 126-143.

Motta-Roth, D. (2008) "Para ligar a teoria à prática: roteiro de perguntas para orientar a leitura/análise crítica de gênero. In: Motta-Roth, D.; Cabañas, T.; Hendges, G. R. (Org.). Análise de textos e de discursos: relações entre teorias e práticas, 2.ed. Santa Maria: PPGL - Editores.

Motta-Roth, D; Hendges, G. R. (2010) "Explorando modalidades retóricas sob a perspectiva da multimodalidade". In: Revista Letras, Universidade Federal de Santa Maria, Programa de Pós-Graduação em Letras, v. 20, n. 40, p. 43-66, jan./jun. 2010. 
Oliveira, A.; Fonseca, T. e Biazus, M. (2007) "O uso da Web em produção multimídia na área de artes visuais”. In Informática na Educação: Teoria \& Prática - Revista do PGIE da UFRGS, v. 10, n. 1.

Oliveira, A.; Schaf, F. M.; Reis, S. C. (2012) "LABINTER - Connecting and creating Interdisciplinary interactive e-learning projects". In International Association for Development of the Information Society International Conference e-Learning 2012, Lisboa. Proceedings of the IADIS Multiconference on Computer Science and Information Systems (MCCSIS 2012), p. 455-459.

OpenSim. (2012) “Open Simulator Project Website”. http://opensimulator.org, Março.

Paiva, V. L. M. O. (1999) "Diários online na aprendizagem de língua inglesa mediada por computador". In Mari, H.; Machado, I. L.; Mello, R. (Org.) Fundamentos e dimensões da análise do discurso. Belo Horizonte: Carol Borges: Belo Horizonte, p. 359-378.

Paiva, V. L. M. O. (2010) "Interação e aprendizagem em ambiente virtual". 2 ed. Belo Horizonte: Editora UFMG.

Piaget, J. (1962) "A Linguagem e Pensamento da Criança". Routledge \& Kegan Paul, London, UK.

Reis, S. C. (2004) “A intervenção pedagógica do professor em contextos diferenciados: a oferta de andaimes na aula de inglês presencial e a distância”. Dissertação. Instituto de Estudos da Linguagem - IEL, Unicamp: Campinas, SP.

Reis, S. C. (2005) "O chat como um gênero privilegiado para o ensino colaborativo de língua inglesa”. In Simpósio Internacional de Estudos de Gêneros Textuais (SIGET). Santa Maria, Anais..., Santa Maria: Universidade Federal de Santa Maria, RS, v. 1, n. 1, p. 1-12.

Reis, S. C. (2006) "O bate-papo educacional: um gênero potencial para práticas sociais e atividades pedagógicas a distância”. In Linguagens \& Cidadania, v. 6, p. 1-16. http://jararaca.ufsm.br/websites/l\&c/download/Artigos/L\&C 2S 06/SuzanaL\&C200 6.pdf, Outubro.

Reis, S. C. (2010) "Do discurso à prática: textualização de pesquisas sobre ensino de inglês mediado por computador". Tese de Doutorado. Universidade Federal de Santa Maria, Santa Maria, RS.

Schaf, F. M.; Paladini, S.; Pereira, C. E. (2012) “3D AutoSysLab Prototype - A Social, Immersive and Mixed Reality Approach for Collaborative Learning Environments". In International Journal of Engineering Pedagogy (IJEP), v. 2, p. 15-22.

Vygotsky, L. V. (1998) “A Formação Social da Mente”. Martins Fontes, São Paulo. 